\title{
A big data problem involving stick-slip oscillations in an electromechanical system
}

\author{
Roberta Lima ${ }^{1}$ \\ PUC-Rio, Rio de Janeiro, RJ \\ Rubens Sampaio ${ }^{2}$ \\ PUC-Rio, Rio de Janeiro, RJ
}

\begin{abstract}
This work analyzes the stochastic response of an electromechanical system with stickslip oscillations. The system is composed of two subsystems that interact, a mechanical and an electromagnetic (a DC motor). The mechanical subsystem is subject to a dry-frictional force modeled as a Coulomb friction. An imposed source voltage in the DC motor stochastically excites the system. This excitation induces in the mechanical subsystem stochastic stick-slip oscillations. The resulting motion of the mechanical subsystem can be characterized by two qualitatively different and alternate modes, the stick- and slip-modes, with a non-smooth transition between them. Mechanical and electromagnetic parameters influence this stochastic sequence. The objective of this paper is to analyze the joint influence of these parameters in the statistical model of the system response. With Monte Carlo simulations, scatter plots and joint histograms are constructed for different combinations of parameter values.
\end{abstract}

Key words. Electromechanical systems, Stick-slip oscillations, Dry-friction, Uncertainties, Big data, Joint distributions

\section{Introduction}

The presence of dry-friction in mechanical systems may induce the occurrence of stick-slip oscillations, a type of motion with a non-smooth behavior. When stick-slip occurs, the system response is characterized by two qualitatively different modes, the stick- and slip-modes. We call stick when the relative velocity between the bodies in contact is null in a time-interval of nonnull duration and we call slip if the relative velocity is non-zero, or zero in isolated points. The literature dealing with sick-slip oscillations in mechanical systems is extensive [2]. Nevertheless, this paper brings a novelty. It analyzes the sick-slip oscillations in an electromechanical system. The analyzed system is composed of two subsystems that interact, a mechanical and an electromagnetic (DC motor). The system mode at each instant (stick or slip) depends on the state of the whole system, i.e., depends on mechanical and electromagnetic variables. Usually, stick-slip oscillations appear in systems in which uncertainties play an important role. Dry friction forces present an inherent random behavior. The influence of ambient conditions in the properties of contact surfaces turns the dry friction force uncertain $[1,2]$. The source of uncertainties of the multiphysics system analyzed in this paper are the imposed source voltage in the electromagnetic subsystem and the initial condition of the position of the mechanical subsystem. The excitation induces in the mechanical subsystem stochastic stick-slip oscillations. Mechanical and electromagnetic

\footnotetext{
${ }^{1}$ robertalima@puc-rio.br.

${ }^{2}$ rsampaio@puc-rio.br.
} 
parameters influence this stochastic sequence. The objective of this paper is to analyze the joint influence of these parameters in the statistical model of the system response. In this statistical model, the variables of interest of the sequence are modeled as random variables, as for example, the number of time intervals in which stick or slip occur, the instants at which they begin, and their duration. In this paper, we focus on the stick duration and in the position of the mechanical subsystem during the stick. With Monte Carlo simulations, scatter plots and joint histograms of the stick duration and the position of the mechanical system during the stick were constructed for stick duration for different values of friction coefficient. To construct these joint histograms with accuracy, several realizations of the system response were required. Since each realization was obtained by a numerical integration of the initial value problem that gives the dynamics of the system, the computational cost to perform the simulations was high. The developed analysis belong to a class of big data problems.

This paper is organized as follows. Section 2 presents the dynamics of an electromechanical system with dry-friction, i.e., the initial value problem (IVP) that describes the dynamics of the coupled system. The dry-friction force model, and the necessary conditions for the occurrence of the stick- and slip-modes are defined in Section 3. In Section 4, an analytical approximation to the upper bound for the stick duration is presented. The influence of mechanical and electromagnetic variables and parameter values in this upper bound is discussed in Section 5. With the analytical approximation, it is possible to determine the influence of the position of the mechanical system during the stick in the stick duration. The upper bound will define the support of the joint distributions of interest in the paper. The construction of the probabilistic model of the uncertain source voltage is given in Section 6. Scatter plots and joint histograms constructed for stick duration are presented in Section 7.

\section{Dynamics of the electromechanical system with dry-friction}

The system analyzed in this paper is composed by a cart-disk whose motion is driven by a DC motor. The motor is coupled to the cart by a scotch-yoke mechanism that transforms the motor rotational motion into horizontal cart motion over a rail, as shown in Fig. 1. The initial value
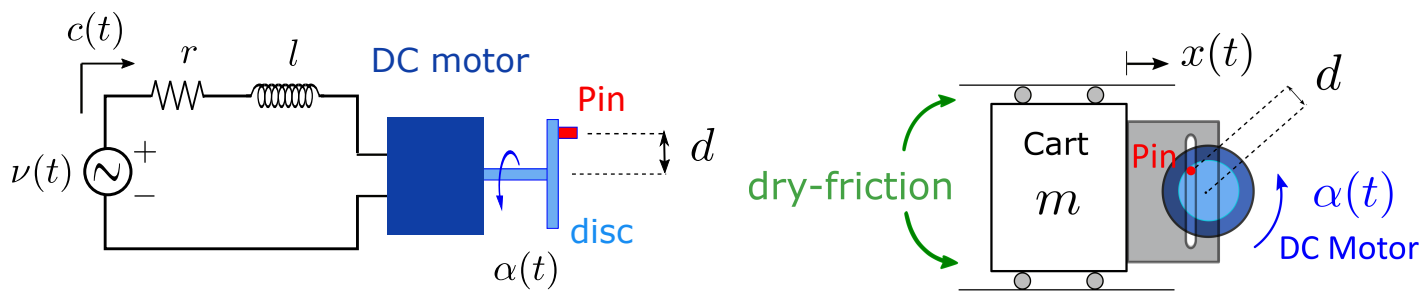

Figure 1: Electromechanical system with dry-friction between the cart and the rail.

problem that describes the system dynamics is given in Eqs. (1) and (2). Find $(\alpha, c)$ such that, for all $t>0$

$$
\begin{gathered}
l \dot{c}(t)+r c(t)+k_{e} \dot{\alpha}(t)=\nu, \\
\ddot{\alpha}(t)\left[j_{m}+m d^{2}(\sin (\alpha(t)))^{2}\right]+\dot{\alpha}(t)\left[b_{m}+m d^{2} \sin (\alpha(t)) \cos (\alpha(t))\right] \\
-k_{e} c(t)=-f_{r}(t) d \sin (\alpha(t)),
\end{gathered}
$$

with the initial conditions $\dot{\alpha}(0)=\beta, \alpha(0)=\alpha_{0}$ and $c(0)=c_{0}$. In Eq. (1) and (2) $t$ is the time, $v$ is the source voltage, $c$ is the electric current, $\dot{\alpha}$ is the angular speed of the motor, $l$ is the electric inductance, $j_{m}$ is the motor moment of inertia, $b_{m}$ is the damping ratio in the transmission of the 
torque generated by the motor, $k_{e}$ is the motor electromagnetic force constant, $r$ is the electrical resistance, $m$ is the mass of the cart, $d$ is the eccentricity of the scotch-yoke mechanism and $f_{r}$ is the dry-friction force between the cart and the rail. The source voltage $\nu$ is considered to be $\nu(t)=\nu_{0}+\nu_{1} \sin \left(\omega_{v} t+\theta\right)$, i.e., the source voltage oscillates around $\nu_{0}$ with amplitude $\nu_{1}$ and phase $\theta$. Please note that the system state is given by three variables, two of them mechanical (angular velocity and position of the motor) and one of them electromagnetic (current). The dynamics of the electromechanical system analyzed is given by an initial value problem comprising a set of two coupled differential equations.

\section{Dry-friction model and necessary conditions for the oc- currence of the stick- and slip-modes}

The friction is modeled as Coulomb's and as simple as possible, Fig. 2(a).

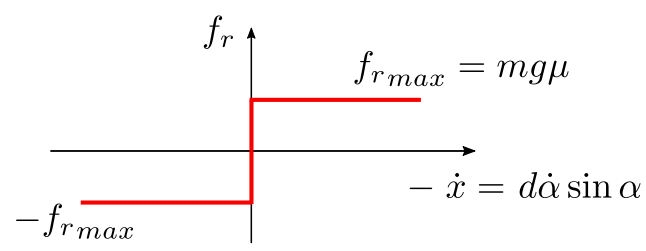

(a)

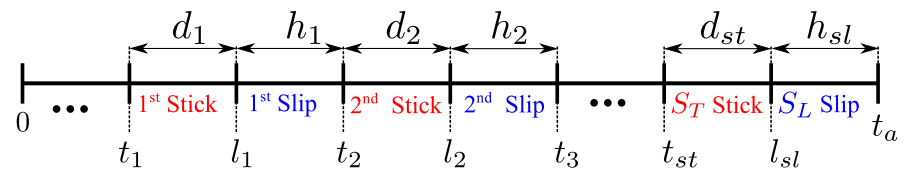

(b)

Figure 2: (a) Coulomb dry-friction and (b) Sequence of stick- and slip-modes in a electromechanical system response.

When the cart velocity is not zero, $\dot{x} \neq 0$, the dry-friction force can only assume the values $-f_{r_{\max }}$ and $f_{r_{\max }}$. However, when $\dot{x}=0$, it can assume any value in the interval $\left[-f_{r_{\max }}, f_{r_{\max }}\right]$. It is considered $f_{r_{\max }}=\mu \mathrm{mg}$, where $g$ is the gravity and $\mu$ is the friction coefficient between the cart and the rail. Note that the dry-friction force is not a function of the cart velocity. For one value of cart velocity, $\dot{x}=0$, the friction can take infinite values, but it is confined in an interval, i.e., its magnitude is bounded. Depending on the values of the system parameters, the response of the system may be composed of a sequence alternating stick- and slip-modes, as illustrated in Fig. 2(b). During a stick-mode, the disk-cart does not move, so that the angle, describing the angular position of the disk, is constant. The frictional force and the current, however, can vary. Hence, stick means only no motion of the disk-cart, the mechanical subsystem. The electromagnetic subsystem continues to change its state until it gathers enough power to move the disk-cart again. The stickmode occurs when $\dot{\alpha}=0$ in an time interval and the frictional force is the interval $\left[-f_{r_{\max }}, f_{r_{\max }}\right]$. To better understand the effect of the stick-mode in the dynamics of the electromechanical system, let us investigate how it influences the IVP that describes the system dynamics. Considering $\dot{\alpha}=0$ and $\ddot{\alpha}=0$ in Eqs. (1) and (2), the following equations are obtained

$$
\begin{gathered}
l \dot{c}(t)+r c(t)=v_{0}+v_{1} \sin \left(\omega_{v} t\right), \\
k_{e} c(t)=f_{r}(t) d \sin (\alpha(t)) .
\end{gathered}
$$


Observe that the first differential equation of the IVP becomes a differential equation (Eq. (3)) which just depends on the current, an electromagnetic variable. The second differential equation of the IVP becomes an algebraic equation (Eqs. (4)). During the stick-mode, the coupling between the electromagnetic and mechanical subsystems is made by Eq. (4). The value of the friction force, during the stick-mode, can vary and follows Eqs. (4), i.e., it depends on an electromagnetic variable and on the angular position of the motor, a mechanical variable. Remark that during the stick-mode, the sum of the forces that act over the cart is zero (it does not move). The horizontal coupling force between the DC motor and the cart, $f$, is balanced by the dry-friction force, $f_{r}$. This balance lasts until the frictional force, given in Eq. (4), reaches its maximum value, $f_{r_{\max }}$. Recall that when $\dot{x}(t)=0$, the magnitude of the frictional force is bounded. During the stick-mode, the dynamics of the system is governed only by the dynamics of the electrical circuit of the motor, the electromagnetic subsystem. During the slip-mode, the dry-friction force is

$$
f_{r}(t)=-m g \mu \operatorname{sgn}(\dot{x}(t))=-m g \mu \operatorname{sgn}(-\dot{\alpha}(t) d \sin (\alpha(t)))
$$

\section{Approximation to the upper bound for the stick duration}

To obtain an analytical approximation to the upper bound for the stick duration, the starting point is the initial-value problem that describes the dynamics of the coupled motor-disk-cart system with dry-friction during the stick-phase given by Eq. (3). Calling the instant of beginning of a stick as $t_{b}$; the instant of end of a stick as $t_{e}$; the angle of the disk in the beginning of a stick, the angle where the disk is stuck, as $\alpha\left(t_{b}\right)=\alpha^{\star}$; and the current in the beginning of a stick as $c\left(t_{b}\right)=c_{0}$. The stick-mode $\left(t_{b} \leq t \leq t_{e}\right)$ is governed by the following IVP:

$$
\begin{aligned}
& l \dot{c}(t)+r c(t)=\nu_{0}+\nu_{1} \sin \left(\omega_{v} t\right) . \\
& c\left(t_{b}\right)=c_{0}
\end{aligned}
$$

The analytical solution of this $\operatorname{IVP}\left(t_{b} \leq t \leq t_{e}\right)$ is given by

$$
c(t)=\left[\frac{c_{0}-\frac{\nu_{0}}{r}-\frac{\nu_{1} \sqrt{r^{2}+l^{2}}}{l^{2} \omega_{v}^{2}+r^{2}} \sin \left(\omega_{v} t_{b}+\phi\right)}{e^{-r / l t_{b}}}\right] e^{-r / l t}+\frac{\nu_{1} \sqrt{r^{2}+l^{2}}}{l^{2} \omega_{v}^{2}+r^{2}} \sin \left(\omega_{v} t+\phi\right)+\frac{\nu_{0}}{r},
$$

where $\phi=\arctan \frac{-l}{r}$. Since, the variable of interest is the upper bound for the stick duration, the influence of the initial condition $\left(c_{0}\right)$ can be neglected. Thus, the solution of the IVP during the the stick-mode $\left(t_{b} \leq t \leq t_{e}\right)$ can be approximated by:

$$
c(t) \approx \frac{\nu_{1} \sqrt{r^{2}+l^{2}}}{l^{2} \omega_{v}^{2}+r^{2}} \sin \left(\omega_{v} t+\phi\right)+\frac{\nu_{0}}{r} .
$$

Recalling that during the stick-mode $f_{r} \in\left[-f_{r_{\max }}, f_{r_{\max }}\right]$ and $k_{e} c(t)=f_{r}(t) d \sin \alpha(t)$. The stick ends when $c\left(t_{e}\right)=\frac{f_{r_{\max }} d \sin \alpha^{\star}}{k_{e}}$. The upper bound for the stick duration $t_{e}-t_{b}$ is the maximal interval during which the current, given by Eq. (8), is lower than the value $\frac{f_{r_{\max }} d \sin \alpha^{\star}}{k_{e}}$. Please observe that if $c$ is always under this value, the stick can last forever. If $c$ is always above, the stick does not happen. 


\section{Influence of mechanical and electromagnetic variables in the obtained approximation}

The upper bound for the stick duration depends on mechanical and electrical variables and geometry of the stick. Among all variables, two of them can be highlighted. They are the angle of the disk in the beginning of a stick, $\alpha\left(t_{1}\right)=\alpha^{\star}$, and the value of the friction coefficient, $\mu$. To better visualize the influence of these two variables, the approximation to the upper bound for the stick duration was computed for different combinations of $\alpha^{\star}$ and $\mu$. The values to all the others parameters are fixed as $l=1.880 \times 10^{-4} \mathrm{H}, j_{m}=1.210 \times 10^{-4} \mathrm{~kg} \mathrm{~m}{ }^{2}, b_{m}=1.545 \times 10^{-4} \mathrm{Nm} /(\mathrm{rad} / \mathrm{s})$, $\nu_{0}=1.000 \mathrm{~V}, \nu_{1}=0.500 \mathrm{~V}, \omega_{v}=10.000 \mathrm{rad} / \mathrm{s}, k_{e}=5.330 \times 10^{-2} \mathrm{~V} /(\mathrm{rad} / \mathrm{s}), r=0.307 \Omega$, $m=5.000 \mathrm{~kg}, d=0.010 \mathrm{~m}$. The results are shown in Figs. 3(a) and 3(b). In Fig. 3(a), the values of friction coefficient are $0.1,0.2,0.3,0.4,0.5$. It can be observed that with $\mu=0.1$, the approximation to the upper bound for the stick duration is 0 for all $\alpha^{\star}$, which means that there is no stick. When $\mu=0.2$, that is possible to have stick. The longest stick happens when $\alpha^{\star}=\pi / 2$ or $\alpha^{\star}=3 \pi / 2$. In the region that $\alpha^{\star}$ is around 0 or $\pi$, there is no stick. As the friction coefficient increases, the region where there is no stick shrinks. In Fig. 3(b), the values of friction coefficient are $0.6,0.7,0.8,0.9,1.0$. There are regions of $\alpha^{\star}$ around $\pi / 2$ and $3 \pi / 2$ that the stick can last forever. This means that if a stick happens when $\alpha^{\star}$ is in a certain region, the stick will last forever.

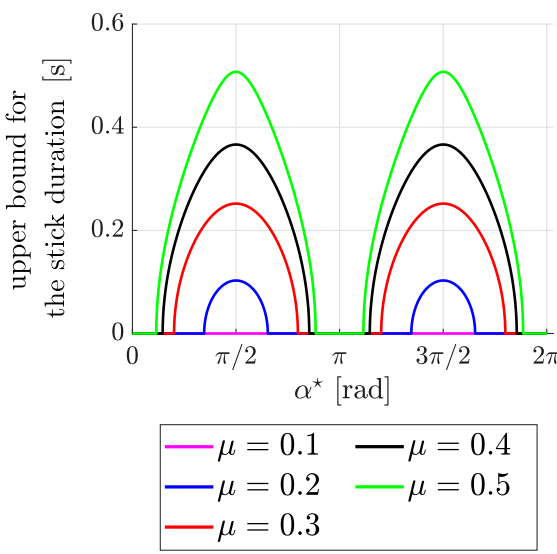

(a)

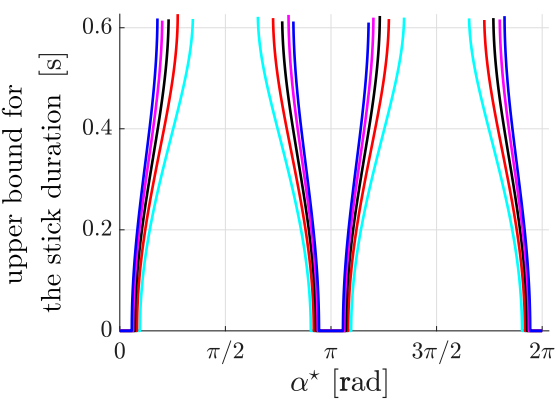

$$
\begin{aligned}
-\mu & =0.6-\mu \doteq 0.9 \\
-\mu & =0.7-\mu=1.0 \\
-\mu & =08
\end{aligned}
$$

(b)

Figure 3: Approximation to the upper bound for the stick duration as function of the angle of the disk in the beginning of a stick for different values of the friction coefficient.

\section{Construction of the probabilistic models}

We consider the source voltage and the initial condition of the angular position of the disk as sources of uncertainties in the stick-slip oscillator problem. We model the phase of the source voltage and the initial condition of the angular position of the disk as uniform random variables over $[0,2 \pi]$. Due to the consideration of uncertainties, the equations of motion of the system, became a stochastic differential equations. The response of the stochastic stick-slip oscillator becomes stochastic and involves two random processes: the angle of the disk and the current. The angle of the disk presents a sequence alternating stick and slip-modes. We are interested in the 
stochastic characterization of this sequence. Defined a time interval for analysis, the variables of interest are the number of time intervals in which stick or slip occur, the instants at which they start, and their duration. These variables are modeled as stochastic objects in order to allow the stochastic characterization the dynamics of the oscillator. In this paper, we focus on the position of the mechanical system (disk) and duration of the first stick. The objective is to determine the joint distribution of these variables.

\section{Scatter plots and joint histograms for stick duration}

With Monte Carlo simulations, scatter plots and joint histograms of the duration and the position of the disk during the first stick were constructed for two different values of friction coefficient, $\mu=0.2$ and $\mu=0.5$. For each value of $\mu$, the initial value problem that characterizes the system dynamics were numerically integrated 10,000 times, totalizing 20,000 numerical integrations. To perform Monte Carlo simulations in a system with stick-slip and uncertainties belong to a class of big data problems. The combination of dry friction and uncertainties makes simulations costly since both involve convergence analysis. In the Monte Carlo simulations, a large number of realizations of the system response was necessary in order to construct the histograms with accuracy [4].It is important to remark that the number of realizations required to construct a joint histogram with accuracy is much higher than the number of realizations required to construct a marginal one. This number should be determined after a convergence study in a two dimension surface. Besides that, stick-slip is a type of dynamics with non-smooth behavior. Thus, when performing the numerical integration of the initial value problem that characterizes the system dynamics, at each time-step, ís necessary to determine in which mode the system is (stick or slip). To accurately predict the switching instants between modes, a small time-step must be used. In this paper, each realization in the Monte Carlo simulations was integrated with a time-step of $10^{-4}$ s. The computational and temporal cost to perform the simulations was high. The CPU time was approximately 30 hours and the amount of data generated was around $20 \mathrm{~GB}$. It is worth noting that in the studied problem, we can generate a database as large as we wish just increasing the number of simulations and reducing the time-step. In our problem, big data can be as big as we want. The limiting factors are processing speed and storage capacity. The parameter values used in all simulations are listed in Section 5. Figures 4 and 5 show the scatter plots and normalized joint histograms of the duration $\left(d_{1}\right)$ and the position $\left(\alpha_{1}^{\star}\right)$ of the mechanical subsystem during the first stick for the different values of friction coefficient. In the scatter plots, it is also shown the approximation to the upper bound for the stick duration as function of the position of the disk during the stick. Please remark that the upper bounds defines the supports of the joint distributions of the duration and the position of the disk during a stick. The non-square support of the joint distributions highlight the stochastic dependence between the two random variables.

\section{Conclusions}

This article analyzes the dynamics of an electromechanical system with dry-friction. The source of uncertainties of the system are the imposed source voltage in the DC motor and the initial condition of $\alpha$. The excitation induces in the mechanical subsystem stochastic stick-slip oscillations. Mechanical and electromagnetic parameters influence this stochastic sequence. The objective of this paper is to analyze the joint influence of these parameters in the statistical model of the system response. Scatter plots and joint histograms of stick duration and the position of the disk during the stick were constructed for different values of friction coefficient. 

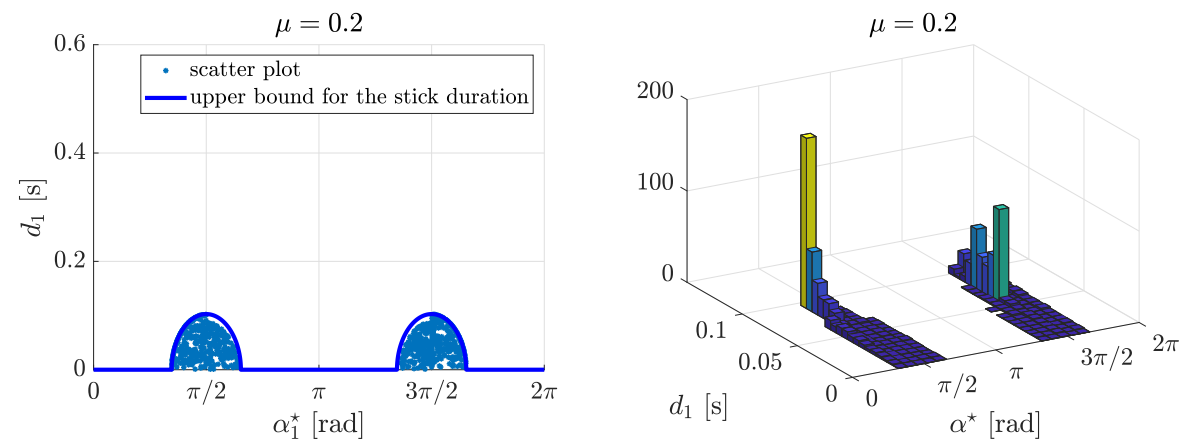

Figure 4: Scatter plots and normalized joint histograms of the duration and the position of the mechanical system of the first stick for $\mu=0.2$.
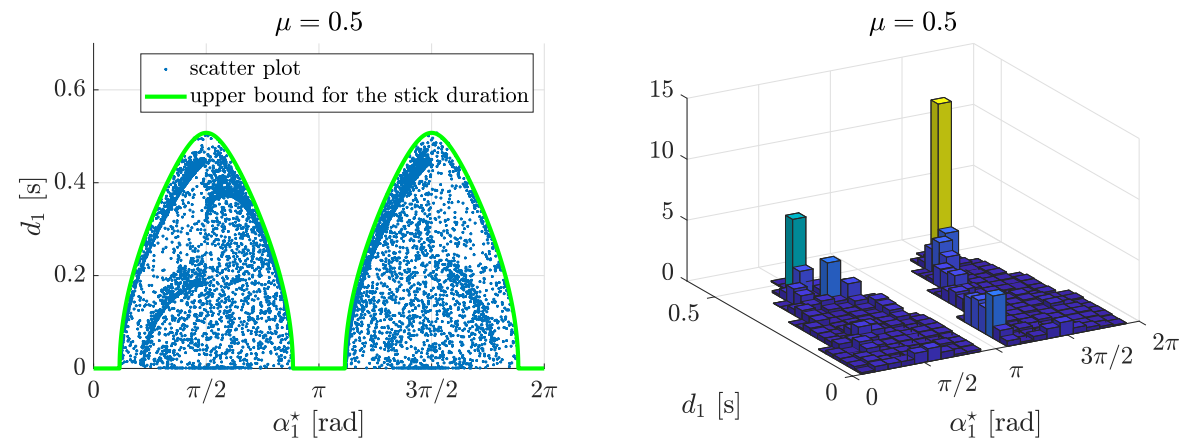

Figure 5: Scatter plots and normalized joint histograms of the duration and the position of the mechanical system of the first stick for $\mu=0.5$.

\section{Acknowledgments}

The authors acknowledge the support given by FAPERJ, CNPq and CAPES.

\section{References}

[1] Bengisu, M.T. and Akay, A. Stick-slip oscillations: dynamics of friction and surface roughness, Journal of the Acoustical Society of America, 105:194, 1999. DOI: 10.1121/1.424580.

[2] Lima, R. and Sampaio, R. Construction of a statistical model for the dynamics of a basedriven stick-slip oscillator, Mechanical Systems and Signal Processing, 91:157-166, 2017. DOI: 10.1016/j.ymssp.2016.12.038.

[3] Lima, R. and Sampaio, R. Stick-slip oscillations in a multiphysics system, Nonlinear Dynamics, 100:2215-2224, 2020. DOI: 10.1007/s11071-020-05677-5.

[4] Sampaio, R. and Lima, R. Modelagem Estocástica e Geração de Amostras de Variáveis e Vetores Aleatórios. In Notas de Matemática Aplicada, vol. 70, SBMAC, São Carlos, São Paulo, 2012. ISSN: 2175-3385. 